\title{
Studying of Volumetric Expansion and Uniaxial Stress-Strain Using Speckle
}

\author{
N. Farid ${ }^{1 *}$, H. Hussein ${ }^{2}$, A. Eltawil ${ }^{3}$, M. Bahrawi ${ }^{1}$ \\ 1.Laboratory of End and Line Standards, National Institute for standards (NIS), Tersa St. Haram, \\ Code:12211, P.O.Box: 136 Giza, Egypt. \\ 2. Primary Length Standard \& Laser Technology Laboratory, National Institute for standards (NIS), Tersa St. \\ Haram, Code:12211, P.O.Box: 136 Giza, Egypt. \\ 3. Laboratory of Mass, Density and Pressures, National Institute for standards(NIS), Tersa St. Haram, \\ Code:12211, P.O.Box: 136 Giza, Egypt. \\ $\left.{ }^{*}\right)$ E-mail: niveen_farid@hotmail.com
}

Received: 06/05/2015 Accepted: 05/11/2015

DOI: $10.7149 /$ OPA.48.4.291

\begin{abstract}
:
The paper presents a speckle photographic technique for studying the effect of both temperature and mechanical stress on a sheet of acrylic (Poly Methyl Methacrylate). A temperature control system was used to heat the acrylic sheet with a homogeneous distribution of temperature on the acrylic surface. Standard masses calibrated at NIS laboratory of mass were used to produce uniaxial stress on the acrylic sheet. The object sides were illuminated by diode laser and the speckle was recorded under both effects and then analyzed by the Fourier transformation. The correlated speckle patterns produced Young's interference fringes which differed in numbers, separation, and orientation according to the applied effect. Both volumetric thermal expansion and modulus of elasticity could be measured and were found to be in good agreement with the corresponding reference values of the object material. Theoretical analysis, method, and results are also presented. The results proved the reliability of the used technique for studying thermal and mechanical expansions of the acrylic material.
\end{abstract}

Key words: Thermal expansion, Stress-Strain, Speckle, Fourier transform.

\section{REFERENCES AND LINKS}

[1] G. Gloud, Optical Methods in Engineering Analyses. Cambridge, Cambridge University Press (1998).

[2] I. Razumovsky, Interference Optical Methods of Deformable Solid Mechanics. Moscow Higher Technical University n.a. N.E. Bauman Publishing House (2007).

[3] A. Chernyatin, I. Razumovsky, "Experimental and Computational Method for Determining Parameters of Stress-Strain State from the Data Obtainable by Interference Optical Techniques," EPJ Web of Conferences 6, 45003 (2010). http://dx.doi.org/10.1051/epjconf/20100645003

[4] M. Abdel Hady, M Necklawi, A. Fahim, M. Bahrawi, N. Farid, "Speckle Photography in Measuring Thermal Expansion," MAPAN 27, 133-137(2012). http://dx.doi.org/10.1007/s12647-012-0025-x

[5] K. Stetson, Fringe 2009: Digital Fourier-transform processing for analysis of speckle photographs, W. Osten, M. Kujawinska eds., Springer - Verlag Berlin Heidelberg (2009). 
[6] F. Chiang, L. Mao, "Development of interior strain measurement techniques using random speckle patterns," Meccanica 50, 401-410 (2015). http://dx.doi.org/10.1007/s11012-014-0048-1

[7] Organization International De MetrologieLegale, “OIML R 111-1", Ed (2004).

[8] N. Farid, H. Hussein, M. Bahrawi, "Employing of Diode Lasers in Speckle Photography and Application of FFT in Measurements," MAPAN 30, 125-129 (2015). http://dx.doi.org/10.1007/s12647-014-0128-7

[9] L. Shirley, G. Hallerman, "Nonconventional 3D Imaging Using Wavelength-Dependent Speckle," Linc. Lab. J. 90, 153-186 (1996).

[10] M. Abdel Hady, Application of multiwavelength speckle in static and dynamic displacements measurement, Master Thesis, Faculty of Science, Helwan university (2013).

[11] J. Goodman, Introduction to Fourier optics, Singapore, $2^{\text {nd }}$ ed: McGraw hill, 63-90 (1996).

[12] B. Zadlera, J. Scales, "Monitoring crack-induced changes in elasticity with resonant spectroscopy," Journal of Applied Physics 104, 1-4 (2008). http://dx.doi.org/10.1063/1.2956688

[13] http://webhotel2.tut.fi/projects/caeds/tekstit/plastics/plastics_PMMA.pdf

\section{Introduction}

Optical methods used in analyzing surface perturbation that could be applied by thermal or mechanical effects provided accurate information on the changes of the surface of interest as well as the value and the direction of the factors causing the change. Speckle technique became the most common technique among the optical methods because of its reliability and the capability to measure all scales. Due to this advantage, it could replace the classical mechanical techniques in studying surface roughness, deformation, stress, strain, displacement etc. Its applications were increased by employing the digital processing of the interference fringes and mapping the values of all surface points [1 - 4].

The basic steps of the optical techniques included recording the interference patterns by a high resolution sensor, processing of the recorded patterns using scientific algorithms depending on the desired information and the type of deformation, and finally displaying the results in a suitable manner. The analysis of the interference pattern depended on the correlation between the corresponding speckles before and after the perturbation. The influence of these effects could be defined by the behavior of the speckle patterns.

Because of the efficiency and simplicity of the Fast Fourier Transform in analyzing the speckle pattern displacement in the frequency domain, it could be used in the study instead of speckle pattern trace method [5].

The volumetric (3D) techniques were developed in the field of experimental mechanics for analyzing expansion and stress that the object encountered. The advantages of those techniques were due to the true 3D imaging capabilities despite the complexity in implementation and computation [6]. The proposed technique was an extension of a $2 \mathrm{D}$ speckle technique and the speckle pattern was recorded at two different sides of the objects. That enabled measuring real time deformation in xyz axes on the specimen body.

The specimen used in the experimental work is Acrylic (a Perspex piece $100 \times 40 \times 10 \mathrm{~mm}$ ) due to its importance originating from its various applications in building, construction, and decoration.

The aim of the study was to analyze the volumetric thermal expansion and the distribution of stressstress state in a Perspex piece subjected to uniform heat and uniaxial load respectively using volumetric speckle photography and FFT.

\section{Methodology}

The system used in the experiment is shown in Figure 1. Heating the specimen was implemented by placing it on a copper plate, which was fixed to a thermo-electric heater/cooler (TEC) Peltier element (Figure 2a). The TEC was fitted from the other side to a heat sink. A heat-conducting paste was used to fill any tiny gap that might appear between the copper plate and the specimen to ensure good thermal 
contact. A temperature transducer connected to the temperature controller (AD 590) was placed on the specimen to detect its actual temperature. A feedback circuit of the temperature controller model Thorlabs LDC202C controlled the temperature of the sample by comparing the set value with the measured value from the temperature transducer and placed the sample at any fixed desired temperature within the range from 19 to $45^{\circ} \mathrm{C}$.

The uniaxial loading was implemented by placing different standard masses on the specimen's upper surface (Figure 2b). The used masses were calibrated with the NIS mass scale using the comparison method. In the weighing cycles, if " $A$ " represented weighing the reference mass and " $B$ " represented weighing the test mass then, three cycles of ABBA were applied for each mass calibration in order to eliminate the possible drift in the measurements. The magnetization and susceptibility of the used specimen were measured and found to be less than the specified limits of the standard mass as specified in OIML R 111-1 2004, then it could be assumed that the uncertainty components due to the magnetism of the masses magnetization and susceptibility were negligible. [7]

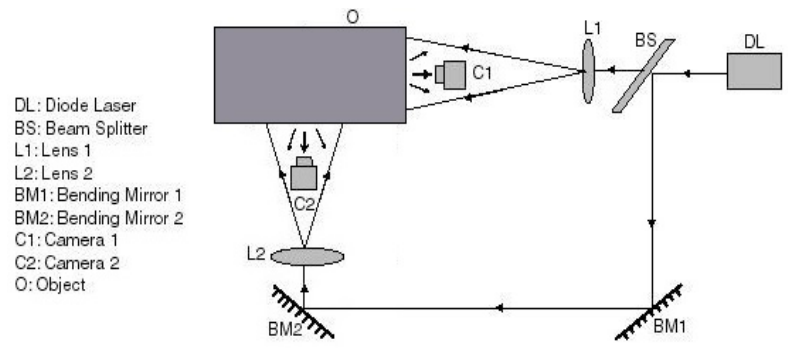

Fig. 1. Setup diagram for recording speckles on the object sides.

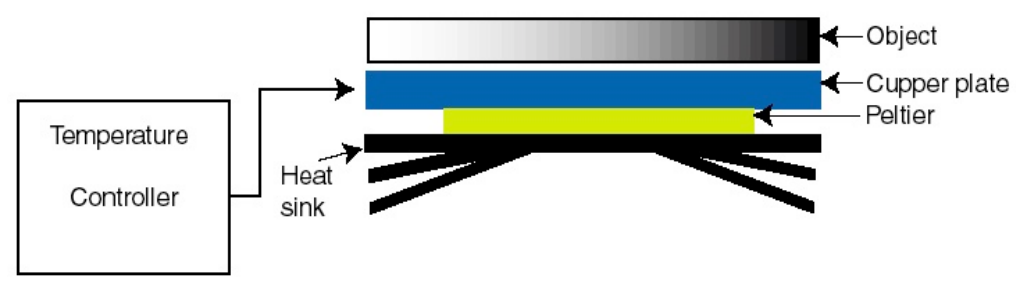

(a) Heating System

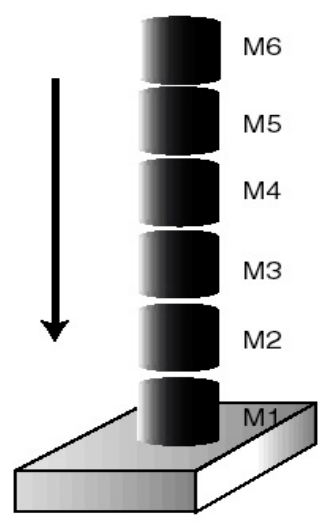

(b) Loads placed on the object

Fig. 2. Diagrams of (a) Heating controlled system and (b) compressive stress applied to the object

Both stress and strain were generated on the object under the effect of the different weights within the range from 0.44 to $2.6 \mathrm{~kg}$. The diode laser was used as a light source that illuminated the object. A suitable wavelength was selected for accurate measurement depending on the roughness degree of the object [8]. 
A suitable power was also selected to keep good visibility of the interference fringes on both sides of the object after beam splitting.

The laser beam was expanded by a beam-expander in order to provide homogenous area of illumination on the object surfaces. The expanded beams were divided into two parts and directed to the object adjacent sides. Two $50 \mathrm{~mm}$ collimating lenses were used to focus the beams scattered by the object sides on the recording sensors of two high-resolution CCD cameras, which are placed $15 \mathrm{~cm}$ from the object sides.

The cameras had powerful optical zoom lenses and the magnification value was selected such that the spatial resolution of the measuring system could be increased. The sensor iris controlled light intensity such that maximum intensity could be used without saturating the camera.

The cameras were connected to two computers where the speckle images on the two object sides were captured and saved.

The speckle patterns on the object sides were recorded during the experiment. The displacement of the speckle patterns defined the micro strains on the specimen's surface, which in turn implied in the separation and orientation of Young's fringes in the FFT domain (Figures 3, 4).

An image processing software was used for handling the captured images and applying the Fourier transformation to the correlated speckle patterns resulted from images combination (i.e. addition). The direction and the spacing of the resulted Young's interference fringes provided information about the orientation and the amount of the object deformation either by heating or loading.

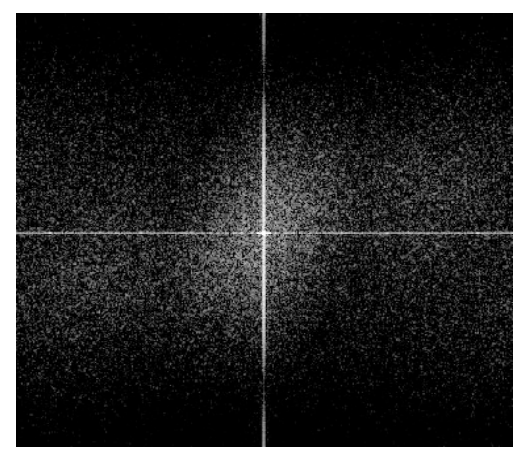

(a)

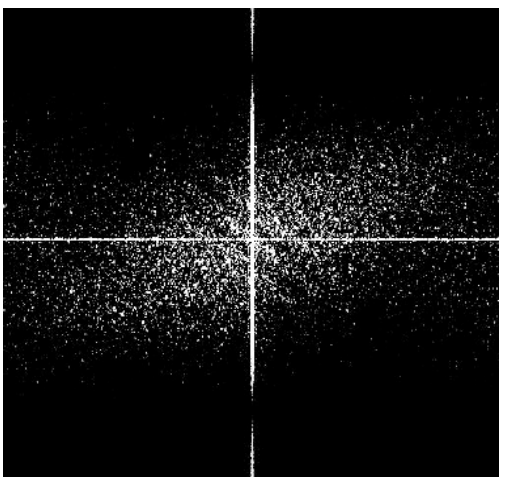

(d)

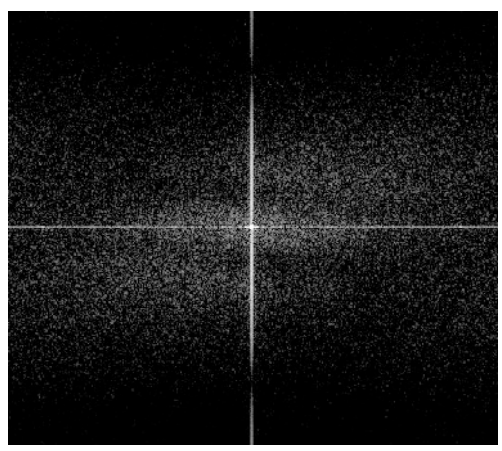

(b)

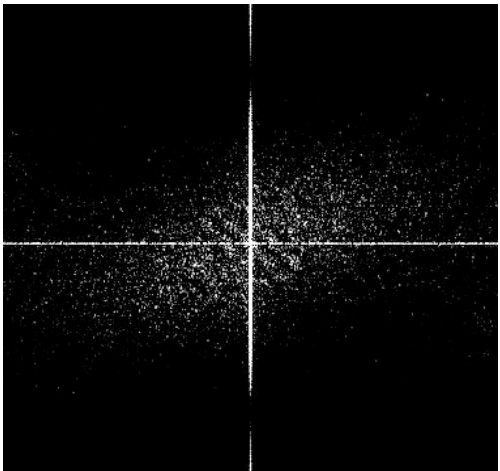

(e)

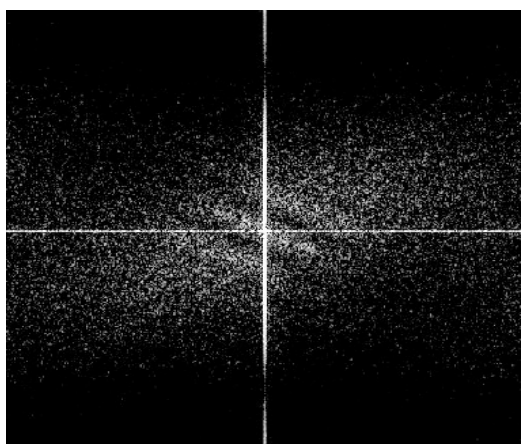

(c)

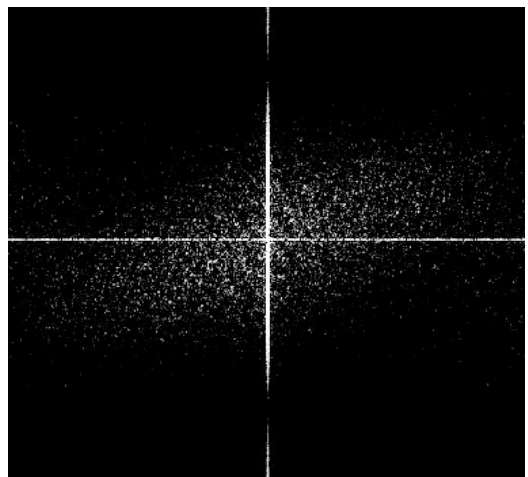

(f)

Fig. 3. FFT of speckle pattern on the object surface at temperatures (a) $19{ }^{\circ} \mathrm{C}$, (b) $25.7{ }^{\circ} \mathrm{C}$, (c) $30.1^{\circ} \mathrm{C}$, (d) $35^{\circ} \mathrm{C}$, (e) $39.5^{\circ} \mathrm{C}$, (f) $44.7^{\circ} \mathrm{C}$. 


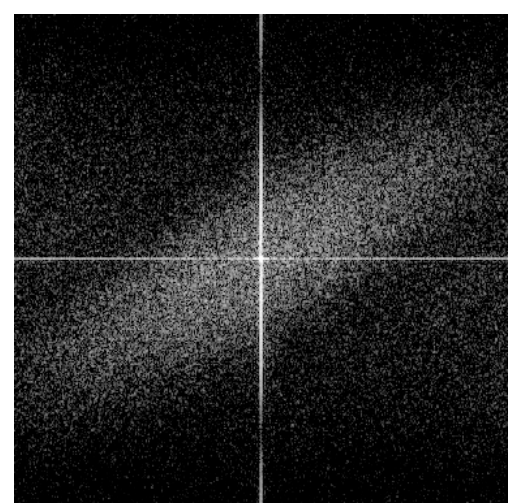

(a)

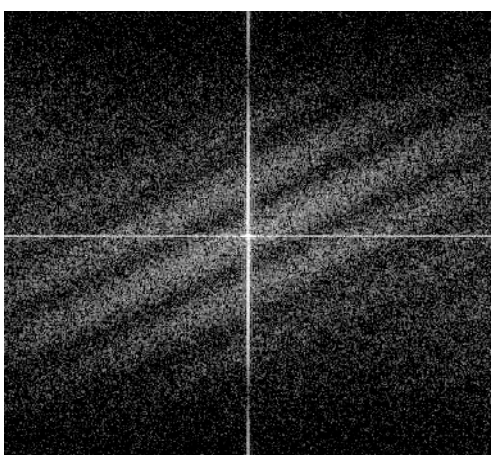

(d)

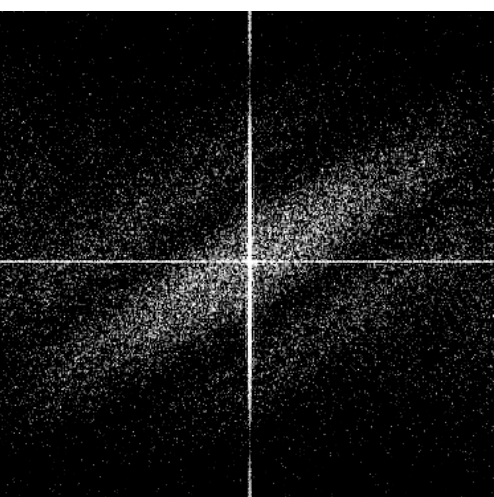

(b)

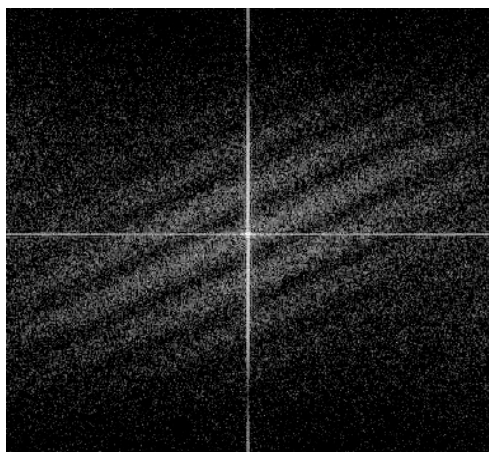

(e)

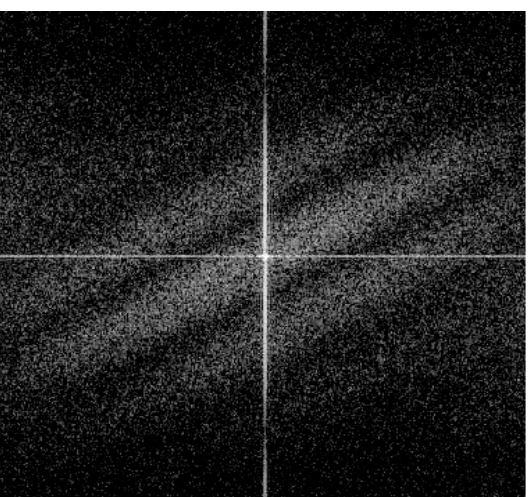

(c)

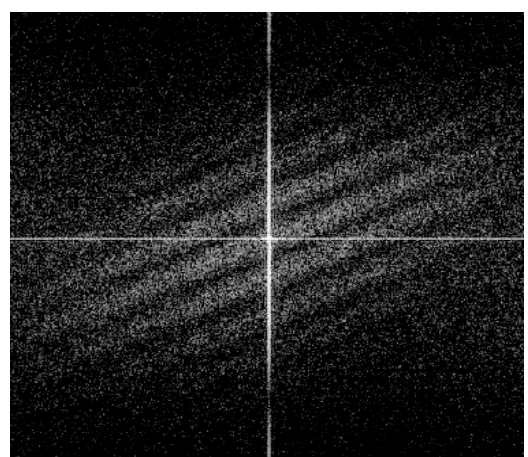

(f)

Fig. 4. FFT of speckle pattern on the object surface under loads (a) $0.44 \mathrm{~kg}$, (b) $0.86 \mathrm{~kg}$, (c) $1.3 \mathrm{~kg}$, (d) $1.7 \mathrm{~kg}$, (e) $2.2 \mathrm{~kg}$, (f) $2.6 \mathrm{~kg}$.

\section{Theoretical aspects}

Assuming the point $O(x, y)$ on the object surface (Side 1) will be displaced to $O^{\prime}(x+\Delta x, y+\Delta y)$ due to heating or loading.

Let $u$ and $v$ be the coordinates in the image plane and they are proportional to the applied thermal and mechanical effects (figure 5).

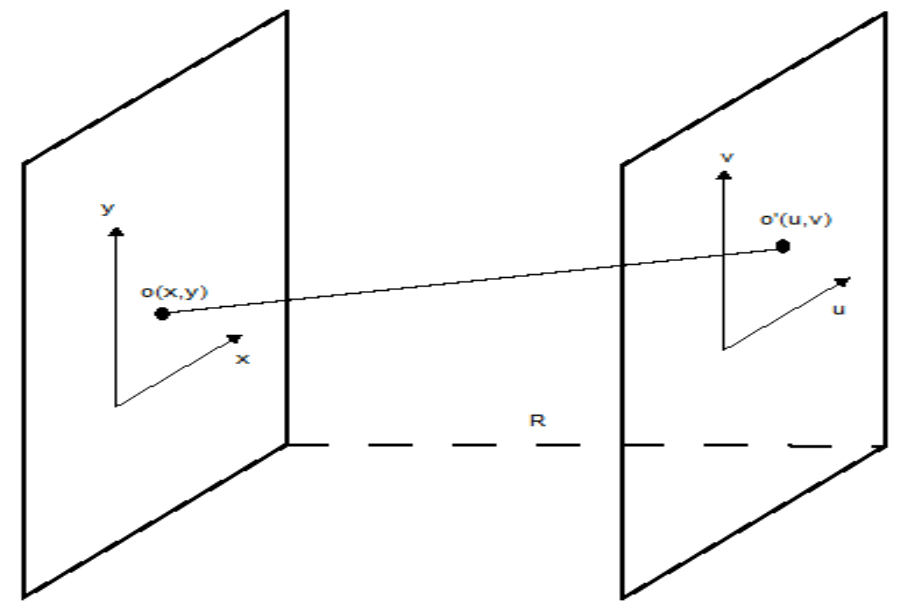

Fig. 5. Diagram of the scattering system

According to [9-11], the complex amplitude at the image plane before the external disturber is given by:

$$
A(u, v)=e^{-j \frac{2 \pi}{\lambda} R} \iint A(x, y) e^{-j 2 \pi\left(x\left(\frac{u}{\lambda R}\right)+y\left(\frac{v}{\lambda R}\right)\right)} d x d y
$$


After either heating effect or mechanical stress, the complex amplitude becomes:

$$
A^{\prime}(u, v)=e^{-j \frac{2 \pi}{\lambda} R} \iint A(x, y) e^{-j 2 \pi\left((x+\Delta x)\left(\frac{u}{\lambda R}\right)+(y+\Delta y)\left(\frac{v}{\lambda R}\right)\right)} d x d y
$$

Where

$(x, y)$ and $(u, v)$ are the coordinates in object and image planes respectively.

$\Delta x$ and $\Delta y$ are the displacement in the object plane resulted by either heating or loading.

$R$ is the separation between object plane and image plane.

$D$ is the distance between the object point and its image.

$\lambda$ is the wavelength used in illuminating the object $(\lambda=780 \mathrm{~nm})$.

The spatial frequencies are defined by:

$$
f_{u}=\frac{u}{\lambda R} ; \text { and } f_{v}=\frac{v}{\lambda R}
$$

Total intensity becomes:

$$
\begin{gathered}
I=\left|A(u, v)+A^{\prime}(u, v)\right|^{2} ; \\
I=2 F T A(x, y)^{2}\left(1+\cos 2 \pi\left(\Delta x f_{u}+\Delta y f_{v}\right)\right)
\end{gathered}
$$

Maximum intensity is obtained at

$$
\Delta x f_{u}+\Delta y f_{v}=n \text { at } n=0,1,2, \ldots
$$

The formed interference fringes are described by:

$$
v=-\frac{\Delta x}{\Delta y} u+\frac{n \lambda R}{\Delta y}
$$

at $v=0$ :

$$
u=\frac{n \lambda R}{\Delta x}
$$

at $u=0$ :

$$
v=\frac{n \lambda R}{\Delta y}
$$

Assume $\Delta u$ and $\Delta v$ are the spatial period of the fringes in the frequency domain.

$$
\Delta x=\frac{\lambda R}{\Delta u} \text { and } \Delta y=\frac{\lambda R}{\Delta v}
$$

Similarly the expansion $\Delta z$ in the z-axis could be determined from the other side of the object (Side 2):

$$
\Delta z=\frac{\lambda R}{\Delta w}
$$

where $\Delta w$ is the displacement in the image plane corresponding to $\Delta z$.

\section{In case of thermal expansion measurement:}

Since the temperature control system provides uniform temperature distribution on the object surface, then the thermal expansion coefficients can be described as:

$$
\alpha_{x}=\frac{\Delta x}{X} \cdot \frac{1}{\Delta T} ; \quad \alpha_{y}=\frac{\Delta y}{Y} \cdot \frac{1}{\Delta T} ; \alpha_{z}=\frac{\Delta z}{Z} \cdot \frac{1}{\Delta T} ;
$$

and the volumetric thermal expansion is represented by

$$
\alpha_{v}=\frac{d v}{V} \cdot \frac{1}{\Delta T}
$$


According to [12], the volumetric thermal expansion of isotropic material is relevant to the linear thermal expansion such that:

where $\alpha_{l}$ is the linear thermal expansion.

$$
\alpha_{v}=\frac{d v}{V} \cdot \frac{1}{\Delta T}=3 \alpha_{l}
$$

In case of stress-strain measurement:

The strain of both object sides $\mathcal{E}$ under compressive stress can be defined as:

$$
\varepsilon=\frac{\Delta L}{L}=\frac{\Delta x}{X}=\frac{\Delta z}{Z}
$$

where $\Delta L$ is the micro deformation in the length due to the uniaxial stress.

The stress force $\sigma$ affecting on the object will be:

$$
\sigma=E \varepsilon
$$

where $E$ is the modulus of elasticity of the material of interest.

\section{Results and discussion}

A preliminary test was made to calibrate the software for measurement. The object was shifted $100 \mu \mathrm{m}$ by a micro-stage before measurement, and the corresponding displacement represented by the speckle pattern in pixel could be identified in micron. Speckle size was adjusted to adequate the fine micro-strains resulted either by heat or mechanical stress.

Figure 6 shows the relation between the reciprocal of the fringe spacing and the temperature change. According to equation (10), the reciprocal of the fringe spacing is proportional to the displacement in the speckle patterns caused by either heat or stress. The relation in figure 6 indicates linear growth in the object dimensions as the temperature raises. The linear thermal expansion values in the three coordinates are calculated from the slops of the straight lines in the figures according to equation (11) as:

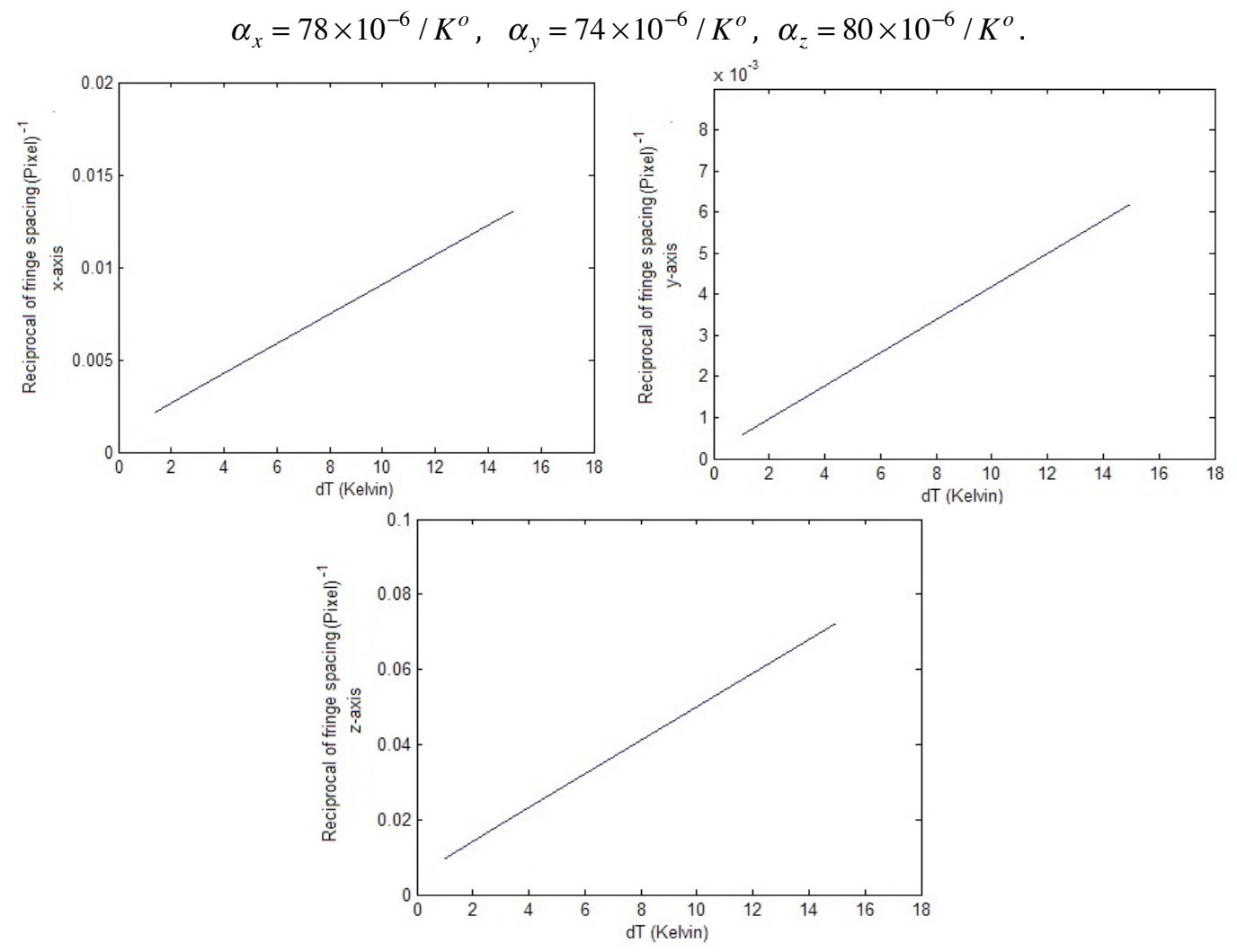

Fig. 6. The reciprocal of fringe spacing versus temperature variation. 
The measured values are compared with the reference value in [13] and they are equal. These values reflect symmetrical growth in the object dimensions with heat. The unfractured acrylic sheet is isotropic and by considering the increase in the specimen volume with temperature, the volumetric thermal expansion can be determined as:

$$
\alpha_{v}=23 \times 10^{-5} / K^{o}
$$

The steel loads applied to the objects generate micro-strains starting from the minimum stress value. The micro-strains increase gradually under load increment. Figure 7 shows the influence of load increment on the object strain. The strain values are determined by the FFT while the strain distribution can be analyzed by speckle correlation technique. The effect of the loads is implied in the fringe separation and orientation in Figure 4, and the values of Modulus of Elasticity are calculated from the relation in (12) as:

$$
E_{1}=3.23 \times 10^{9} \mathrm{~N} / \mathrm{m}^{2} \text { and } E_{2}=3.20 \times 10^{9} \mathrm{~N} / \mathrm{m}^{2}
$$

The measured values are in correspondence with the value in [13]. The stress-strain relation is represented by the curve in Figure 7, while the strain distribution on the object sides is shown in Figure 8.

The plot of stress-strain experimental values on each side was in agreement with the theory of the elastic case.

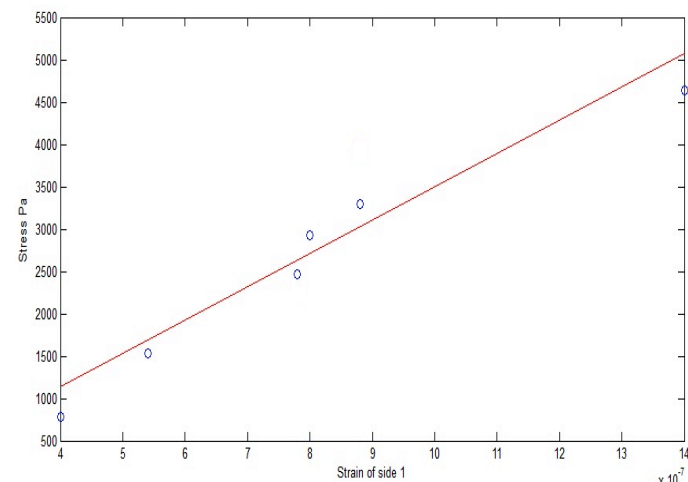

(a)

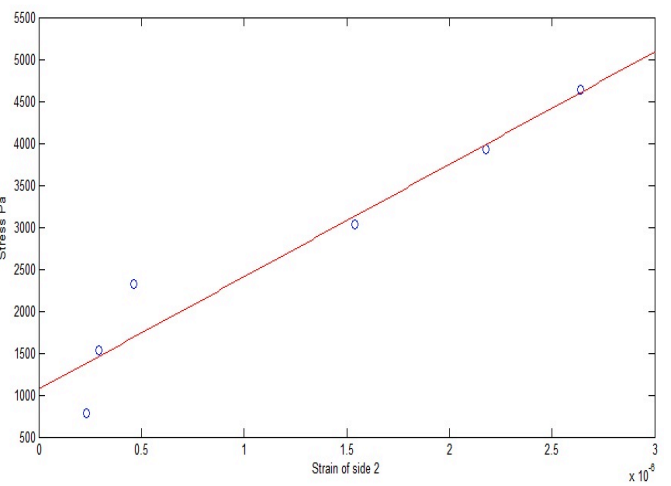

(b)

Fig. 7. The Stress-Strain relation due to compressive stress on (a) Side 1 and (b) Side 2.

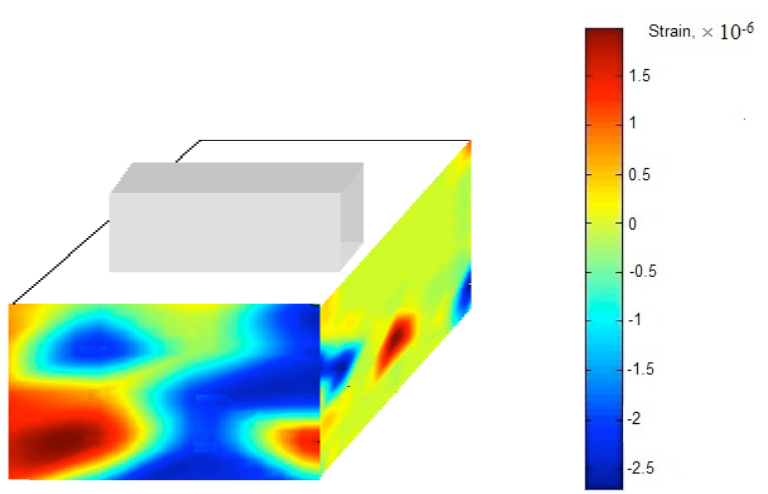

Fig. 8. Strain distribution on the object sides due to compressive stress.

The strain map describes the behavior of both sides of the acrylic sheet under the effect of the loads. Some parts represent tension and others represent compression. Different micro-strains values are observed in nonuniform distribution on both sides. Most of the front side area is exposed to the compressive stress along its dimension and shows faster and higher variation of strain than the other side. However, the strain values on each side are averaged to calculate the modulus of elasticity, and the resultant $E$ values for both sides are similar. 


\section{Conclusions}

Volumetric thermal expansion and stress-strain relation under uniaxial load were studied for a specimen made of acrylic. Speckle photography was used to evaluate the micro level of surface change under both effects and the Fast Fourier transformation was used for analyzing the variation in the object surface implied in Young's fringes separation and orientation. The system was able to provide useful information on the object behavior during the heating and mechanical stress effect. The analysis of the speckle pattern indicated a uniform growth in object dimensions during heating effect. Very small deviations were observed in the calculated linear thermal expansions values which could result from the small shift in time of recording speckle images on each side. The volumetric thermal expansion was found to be $\alpha_{v}=23 \times 10^{-5} / K^{o}$.

The micro-strain resulted from the mechanical stress was studied and the strain distribution on each side was mapped using speckle correlation. Although nonuniform strain distribution on object sides, the avaraged strain values yielded similar values of Modulus of elasticity $\left(E=3.20 \times 10^{9} \mathrm{~N} / \mathrm{m}^{2}\right)$.

The discussed method provided quantitative direct measurement of thermal and mechanical strain that the acrylic sheet may encounter during construction, and the measured values are in agreement with the standard values defined for acrylic material.

The results proved the reliability of the used technique for investigating the strain of the materials, and the major contributors to the accuracy of this method is the resolution of the imaging sensors, the stability of the speckle pattern, and time of recording the speckle images on each side. The simplicity of the system setup enables studying the strain of complex shape objects. 